\title{
4-week daily airway clearance using oscillating positive-end expiratory pressure versus autogenic drainage in bronchiectasis patients: a randomised controlled trial
}

\author{
Galit Livnat ${ }^{1,2,3}$, Naama Yaari ${ }^{4}$, Nili Stein ${ }^{5}$, Lea Bentur ${ }^{3,6}$, Moneera Hanna ${ }^{6}$, Maya Harel ${ }^{7}$, Yochai Adir ${ }^{3,7}$ and \\ Michal Shteinberg (10) $2,3,7$
}

${ }^{1}$ Pediatric Pulmonology, Carmel Medical Center, Haifa, Israel. ${ }^{2}$ Cystic Fibrosis Center, Carmel Medical Center, Haifa, Israel. ${ }^{3}$ Ruth and Bruce Rappaport Faculty of Medicine, Technion-Israel Institute of Technology, Haifa, Israel. ${ }^{4}$ Dept of Physical Therapy, Carmel Medical Center, Haifa, Israel. ${ }^{5}$ Dept of Community Medicine and Epidemiology, Carmel Medical Center, Haifa, Israel. ${ }^{6}$ Pediatric Pulmonology and CF Center, The Ruth Rappaport Children's Hospital, Rambam Medical Center, Haifa, Israel. ${ }^{7}$ Pulmonology Institute, Carmel Medical Center, Haifa, Israel.

Corresponding author: Michal Shteinberg (michalsh@technion.ac.il)

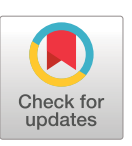

Copyright @The authors 2021

This version is distributed under the terms of the Creative Commons Attribution Non-Commercial Licence 4.0. For commercial reproduction rights and permissions contact permissions@ersnet.org

Received: 26 June 2021 Accepted: 25 Aug 2021

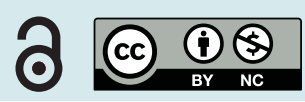

Shareable abstract (@ERSpublications)

People with bronchiectasis were randomised to 4 weeks of two methods of daily airway clearance, autogenic drainage (AD) and oscillating PEP (OPEP). Sputum volume decreased in more people randomised to oPEP than AD. LCl did not change in either group. https://bit.ly/3gNj2bi

Cite this article as: Livnat G, Yaari N, Stein N, et al. 4-week daily airway clearance using oscillating positive-end expiratory pressure versus autogenic drainage in bronchiectasis patients: a randomised controlled trial. ERJ Open Res 2021; 7: 00426-2021 [DOI: 10.1183/23120541.00426-2021].

\section{Abstract}

Background Airway clearance is a fundamental component of bronchiectasis care. Lung clearance index (LCI) is a measurement of ventilation inhomogeneity. Its responsiveness to long-term airway clearance is unknown. We aimed to compare two methods of daily airway clearance over 4 weeks: autogenic drainage (AD) and oscillating positive airway pressure (oPEP), and to determine effects of airway clearance on LCI and clinical outcomes.

Methods Adults with bronchiectasis naive to airway clearance were randomised to daily airway clearance with either $\mathrm{AD}$ or oPEP. Difference in LCI as primary outcome, spirometry, sputum volume and purulence, and quality of life were at randomisation and after 4 weeks of airway clearance.

Results 51 patients (32 women and 19 men, mean age 66.2 \pm 12.8 years) were randomised and 49 completed the study ( $25 \mathrm{AD}$ and $24 \mathrm{oPEP}$ ). The LCI and forced expiratory volume in $1 \mathrm{~s}$ did not change between visits between groups (difference between groups 0.02), nor between visits in either group. Sputum quantity decreased in 12 out of 24 (50\%) of the oPEP group, and in six out of 25 (24\%) of the $\mathrm{AD}$ group $(\mathrm{p}=0.044)$. The "treatment burden" worsened or was unchanged in $70 \%$ of participants randomised to $\mathrm{AD}$ and $55 \%$ randomised to oPEP $(\mathrm{p}=0.038)$.

Conclusion Sputum quantity decreased in more participants randomised to oPEP group after 1 month of daily airway clearance, with a better treatment burden. The effects of 4 weeks of airway clearance on LCI were not significant in either treatment group.

\section{Introduction}

Bronchiectasis is a chronic airway disease characterised by productive cough and bronchial inflammation with abnormal dilatation of the bronchi. Bronchiectasis is associated with poor quality of life and frequent exacerbations [1, 2]. A major component of bronchiectasis is the self-perpetuating process of bronchial infection, inflammation, impaired muco-ciliary clearance and structural lung damage [1]. Therefore, a basic part of bronchiectasis treatment is airway clearance, aimed to prevent mucus stasis [3]. While airway clearance is accepted as a first line treatment, current knowledge on the preferred method is limited. A major obstacle to determining the efficacy of an airway clearance technique is the limitations of currently used end-points [4]. Traditional end-points that have been used include spirometry, exacerbation frequency and quality of life. However, these end-points are relatively insensitive to airway clearance in 
bronchiectasis patients [5, 6]. There is therefore a need to define more sensitive end-points in evaluating interventions to improve airway clearance in bronchiectasis [7].

The lung clearance index (LCI) measured by multiple breath washout (MBW) is a measure of ventilation inhomogeneity and was shown to be sensitive to early ventilation impairment [8]. Its usefulness has been demonstrated in cystic fibrosis (CF), particularly in children and adults with mild disease, with LCI reduction of -0.6 to -1 following therapeutic interventions [9-12]. The LCI has been assessed in bronchiectasis patients and found to be higher (worse) than in healthy subjects, inversely correlating with forced expiratory volume in $1 \mathrm{~s}\left(\mathrm{FEV}_{1}\right)[13,14]$. LCI was found to be non-significantly higher among exacerbating versus stable bronchiectasis patients, and a single session of airway clearance had no effect on LCI [14]. In a study of 60 patients with bronchiectasis, LCI was found to correlate with both radiological severity and $\mathrm{FEV}_{1}$, although LCI was abnormal in more patients than $\mathrm{FEV}_{1}$ [15]. Similar findings were seen in CF patients [16-18].

To the best of our knowledge, no study has been performed to test the long-term effect of any lung clearance technique or device on LCI.

Aerobika (Trudell Medical International, London, Ontario, Canada) [19] is an oscillating positive expiratory pressure (oPEP) device, developed for mobilisation and clearance of secretions in people with suppurative lung diseases. It has been tested and found safe and effective in chronic bronchitis [20, 21]. Autogenic drainage (AD) is a method of airway clearance that aims to propel secretions by controlling the depth of a patient's breathing effort, from low, medium and high breathing volumes, gradually, followed by huffing and expectoration of mucus that finally reaches the larynx [22, 23]. The two methods, AD and oPEP, are commonly used in bronchiectasis; superiority of either one has not been established [24, 25].

The aims of our study were: 1 ) to explore the effects of 4 weeks of airway clearance on LCI; and 2) to compare the effects between two methods of airway clearance, AD and oPEP, on LCI, spirometry, sputum quantity and quality of life in bronchiectasis patients.

Methods

Study population

The study population consisted of patients diagnosed with bronchiectasis, followed at our institution in Carmel Medical Center, Haifa, Israel. Subjects were included if they were $\geqslant 18$ years old, able to give informed consent, had bronchiectasis in at least two lung lobes confirmed by high-resolution computed tomography, were clinically stable, with a predicted $\mathrm{FEV}_{1} \geqslant 50 \%$, and had not regularly performed airway clearance. Patients taking mucolytics, including inhaled isotonic or hypertonic saline, for 3 weeks or more and maintaining the same treatment during the trial were included. Exclusion criteria included a diagnosis of CF or primary ciliary dyskinesia (PCD), and an exacerbation or any change in respiratory medications during the 4 weeks before randomisation. The rationale for excluding people with PCD was that in Israel, a diagnosis of CF or PCD entitles patients to daily physiotherapy treatments by therapists who may not comply with the study protocol. Written informed consent was obtained at inclusion. The study protocol was approved by Carmel Medical Center Helsinki committee (approval no. CMC-87-16) and was registered in ClinicalTrials.gov (trial registration no. NCT03013452).

\section{Study design}

At baseline, patient history was recorded and validated against the participants' electronic medical record of medications, past pulmonary exacerbations (PEx) and quality of life using the quality of life questionnaire for bronchiectasis (QoL-B) [26]. Past pulmonary exacerbations were defined as an increase in at least three respiratory symptoms for 2 days that led to a change in medications [27]. Exacerbations were recorded as separate events if at least 21 days apart. The daily amount of sputum expectorated (e.g. amount in teaspoons, tablespoons or cups) was recorded in equivalent millilitres, and the sputum purulence was quantified according to a validated score on a visual scale [28]. Spirometry and MBW were performed. Participants were randomised by simple randomisation (a random number was generated by an online application for every participant, with even numbers allocated to AD and odd numbers to oPEP) and instructed how to perform the selected method by a study physiotherapist trained in both AD and oPEP. Typical instruction sessions lasted 30 min but were not limited in time. An oPEP device (Aerobika, Trudell Medical International) was provided to patients randomised to this intervention. All participants were instructed to perform the assigned airway clearance technique daily for 15-20 min or until no further sputum was produced, for the duration of the study (4 weeks), and not to perform any other form of airway clearance (this was also specified in a letter to the primary physician given at the first visit). Exacerbations, adverse events and patient-reported adherence to airway clearance were recorded daily by the participants 
and reported weekly by telephone calls. Sputum purulence was assessed by grading on a four-scale visual chart [29]. After 4 weeks, all participants performed an end-of-study visit, which included history and physical examination, review of medications, review of exacerbations since last visit, recording of adverse events and PEx, a repeat QoL-B questionnaire, and performance of MBW and spirometry. The technicians and investigators performing and interpreting spirometry and MBW were blinded to the treatment allocation.

\section{Lung function assessments}

Multiple breath washout measurements were performed using the Easy-One Pro, MBW Module (NDD Medical Technologies, Zurich, Switzerland) as described by Fuchs et al. [30]. The system consists of a side stream ultrasonic transducer for temperature- and humidity-independent sampling of the molar mass, a mainstream ultrasonic transducer for flow sampling and a side stream infrared carbon dioxide $\left(\mathrm{CO}_{2}\right)$ analyser to correct the molar mass signal for exhaled $\mathrm{CO}_{2}$. The WBreath software (NDD) was used for data acquisition, storage and analysis.

The LCI was calculated as the cumulative expired volume during the washout phase divided by the functional residual capacity (FRC), which is the number of FRC turnovers required to wash out nitrogen [31]. An increased LCI indicated more FRC turnovers required for the washout, reflecting ventilation inhomogeneity [8]. As recommended, the minimum re-equilibration time between trials was the time needed for washout in the previous trial [30]. All MBW tests underwent visual quality control before analysis, ensuring regular tidal breathing before washout and absence of a leak.

Spirometry was performed in accordance with the American Thoracic Society/European Respiratory Society (ATS/ERS) Task Force, using a Jaeger MasterScope spirometer (Erich Jaeger AG, Würzburg, Germany) [32].

\section{Statistical analysis}

The primary end-point was defined as the LCI change. Assuming an LCI of 10-12 and a standard error of 0.31-3.4 [13-15], a sample size of 22 subjects in each group was calculated to detect a difference of 1 in LCI with a power of $90 \%$.

Continuous variables are presented as mean \pm SD or medians and interquartile range (IQR). Categorical variables are presented as numbers and proportions. Differences in demographical and clinical characteristics between the two treatment groups (oPEP versus AD) were analysed using the Chi-square test for categorical variables; and independent t-test or Mann-Whitney, as appropriate, for the continuous variables.

Pre/post absolute change and per cent change was calculated. Correlation between two continuous variables (such as $\mathrm{FEV}_{1}$ and LCI) was analysed using the Pearson or Spearman correlation, as appropriate. Paired t-test or Wilcoxon sign rank test was used to check pre/post difference in each group separately for the continuous variables.

Statistical analyses were performed using IBM SPSS Statistics 24.0 (IBM, New York, NY, USA). For all analyses, $\mathrm{p}<0.05$ (for the two-tailed tests) was considered statistically significant.

\section{Results}

A total of 51 patients were recruited between March 2017 and October 2019 (AD group, n=26; oPEP group, $n=25$; figure 1 (Baseline characteristics were similar, except that $\mathrm{FEV}_{1}$ was higher, with more past exacerbations in patients randomised to $\mathrm{AD}$ (table 1).

At baseline, LCI was inversely correlated with $\mathrm{FEV}_{1}$ (correlation coefficient, 0.552; $<<0.0001$ ). Baseline LCI was not correlated with age $(r=0.17, p=0.26)$, sex $(p=0.63)$, PEx in previous 12 months $(p=0.18)$, sputum volume $(\mathrm{r}=-0.005, \mathrm{p}=0.97)$, sputum purulence $(\mathrm{p}=0.412)$, Reiff index $(\mathrm{r}=0.07, \mathrm{p}=0.65)$ or any domain of the QoL-B questionnaire (correlation coefficients for the various domains were in the range of -0.09 to 0.65 ).

Self-reported adherence to airway clearance routine was $88 \pm 2 \%$ and $87 \pm 3 \%$ for the AD and oPEP groups, respectively ( $p=0.63)$. Adverse events were recorded in 13 (59\%) AD participants and 11 (48\%) of the oPEP participants $(p=0.45)$; all were mild and deemed unrelated to treatment. One participant in each group stopped the study due to adverse events (chest pain in one participant performing $\mathrm{AD}$, a pulmonary exacerbation in a participant performing oPEP, both deemed unrelated to the interventions); they were both 


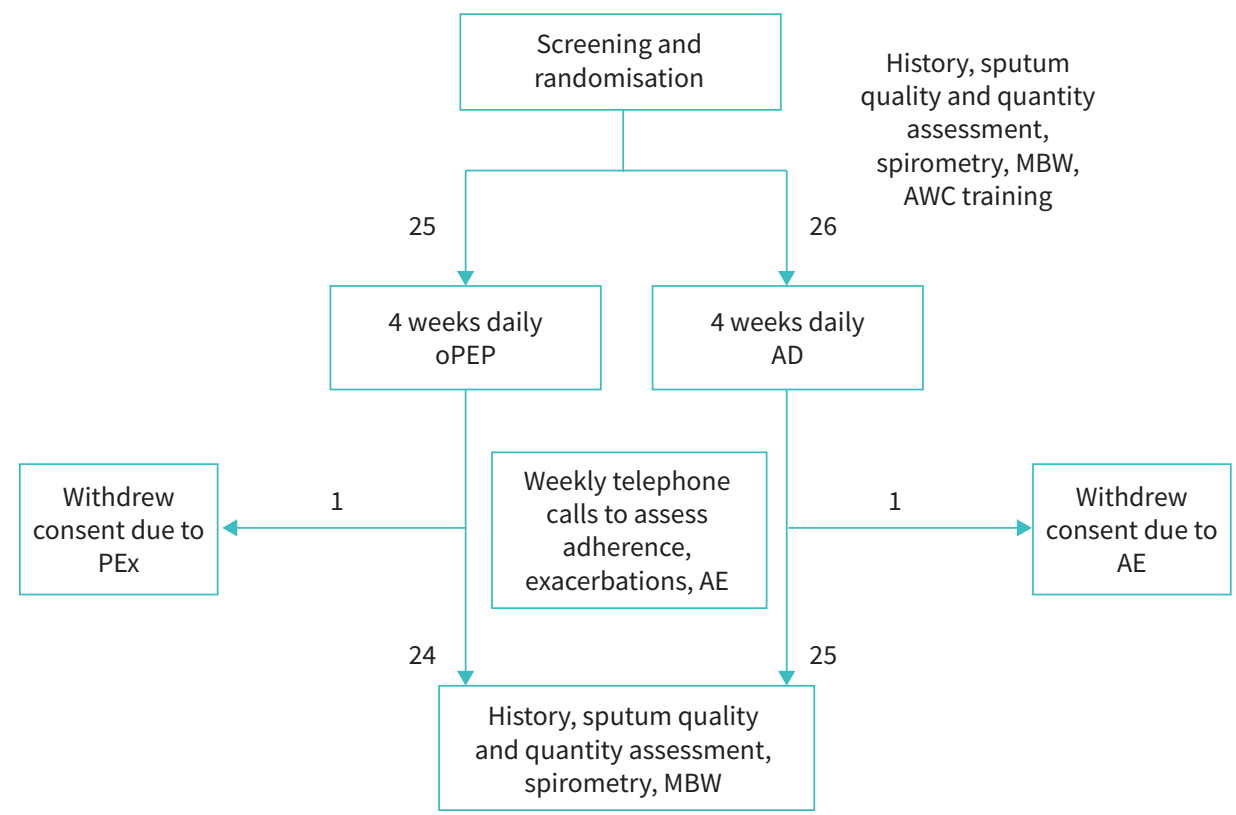

FIGURE 1 Flow of participants through the study. AD: autogenic drainage; AE: adverse event; AWC: airway clearance; MBW: multiple breath washout; OPEP: oscillating positive expiratory pressure; PEx: pulmonary exacerbation.

\section{TABLE 1 Baseline characteristics of patients}

\begin{tabular}{|c|c|c|}
\hline Parameter & $A D$ & OPEP \\
\hline Subjects $\mathrm{n}$ & 26 & 25 \\
\hline Age years (mean \pm sD) & $66.7 \pm 12.3$ & $65.7 \pm 13.4$ \\
\hline Sex (female $n(\%) /$ male $n(\%)$ ) & $19(73.1) / 7(26.9)$ & $14(56.0) / 11(44.0)$ \\
\hline Lung function: $\mathrm{FEV}_{1} \%$ (mean $\left.\pm \mathrm{SD}\right)$ & $96.1 \pm 18.3^{\star \star}$ & $81.3 \pm 18.1^{\star \star}$ \\
\hline Lung function: FVC $\%$ (mean \pm SD) & $101.7 \pm 16.5^{\star}$ & $89.6 \pm 20.0^{\star}$ \\
\hline $\mathrm{LCl}($ mean $\pm \mathrm{sD})$ & $10.0 \pm 2.1$ & $9.0 \pm 2.5$ \\
\hline Daily sputum production $\mathrm{mL}$ (median; IQR) & $17.5(5.0 ; 41.3)$ & $15.0(5.0 ; 20.0)$ \\
\hline Sputum bacterial infection* $\mathrm{n}(\%)$ & $9(34.6)$ & $13(54.2)$ \\
\hline Sputum purulence scale $(0-1 / 2-3) n(\%)$ & $13(59.1) / 9(40.9)$ & $15(62.5) / 9(37.5)$ \\
\hline Previous PEx/year (median, IQR) & $\mathrm{n}=262(1 ; 4)^{\star}$ & $\mathrm{n}=241(0 ; 2)^{\star}$ \\
\hline \multicolumn{3}{|l|}{ Treatment at baseline $\mathrm{n}(\%)$} \\
\hline ICS & $5(19.2)$ & $8(32.0)$ \\
\hline LABA & $5(20.0)$ & $9(36.0)$ \\
\hline LAMA & $0(0)$ & $3(12.0)$ \\
\hline Hypertonic saline & $19(73.1)$ & $20(80.0)$ \\
\hline Performing exercise & $14(53.8)$ & $12(48.0)$ \\
\hline \multicolumn{3}{|l|}{ CT findings } \\
\hline Number of infected lobes on CT (median, IQR) & $\mathrm{n}=212.0(2.0,3.5)$ & $\mathrm{n}=202.0(2.0,3.75)$ \\
\hline Reiff index (median, IQR) & $\mathrm{n}=243.0(2.0,4.0)$ & $\mathrm{n}=253(2,4.5)$ \\
\hline \multicolumn{3}{|l|}{ Findings on auscultation $\mathrm{n}(\%)$} \\
\hline Wheeze & $1(3.8)$ & $3(12.0)$ \\
\hline Rales & $9(34.6)$ & $9(36.0)$ \\
\hline \multicolumn{3}{|c|}{$\begin{array}{l}\text { Sputum bacterial infection relates to any positive culture from a respiratory sample in the past } 12 \text { months. } \\
\text { Sputum purulence was noted on a scale from } 0 \text { to } 3 \text {, with } 0 \text { indicating transparent-yellow and } 3 \text { being green } \\
\text { with traces of blood. AD: autogenic drainage; oPEP: oscillating positive expiratory pressure; SD, standard } \\
\text { deviation; FEV } 1 \text { \%: forced expiratory volume in } 1 \mathrm{~s} \text {, per cent predicted; FVC: forced vital capacity, percent } \\
\text { predicted; LCl: lung clearance index; IQR: interquartile range; PEx: pulmonary exacerbations in the previous } \\
12 \text { months; ICS: inhaled corticosteroids; LABA: long-acting } \beta \text {-agonists; LAMA: long-acting muscarinic agonists; } \\
\text { CT: computed tomography. }{ }^{\star}: \mathrm{p}<0.05 \text {; }{ }^{\star *}: \mathrm{p}<0.001 \text {. }\end{array}$} \\
\hline
\end{tabular}



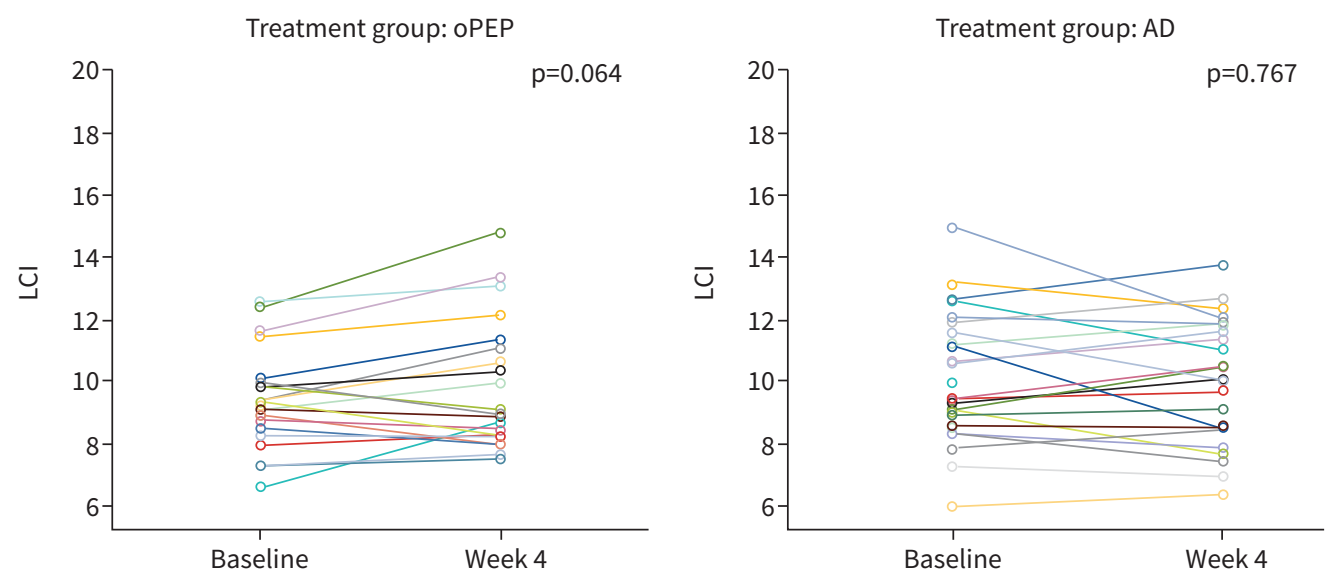

FIGURE 2 Results of lung clearance index (LCI) before and after 4 weeks of daily airway clearance according to method of airway clearance. Each coloured line represents one individual participant. AD: autogenic drainage; OPEP: oscillating positive expiratory pressure.

unavailable for end-of-study assessment, leaving 49 patients eligible for analysis at week 4 (AD, 25; oPEP, 24) (figure 1). In both groups, several participants reported increases in symptoms which necessitated a change in treatment and qualified as a PEx during the study period (six in the AD group; five in the oPEP group).

After 4 weeks of regular airway clearance, there was no significant change in LCI between groups (figure 2): in the oPEP group, LCI changed from mean \pm SD $9.38 \pm 1.6$ to $9.97 \pm 2.5$. In the AD group, LCI changed from $10.04 \pm 2.1$ to $9.88 \pm 2.0$. The difference between groups after 4 weeks was 0.02 . Spirometry parameters did not change in either treatment group (table 2). At 4 weeks, median (IQR) LCI increased by a mean of 0.6 in the oPEP group and decreased by 0.16 in the AD group: mean of difference between groups -0.56 (95\% CI $-1.27-0.21, \mathrm{p}=0.142)$.

Self-reported daily sputum quantity was reduced in 12 (52\%) oPEP participants, compared to 6 (24\%) AD participants after 4 weeks ( $\mathrm{p}=0.04$, table 2). Sputum quantity was reduced from a median (IQR) of $17.5 \mathrm{~mL}(5-42.5)$ to $15 \mathrm{~mL}(5-45)$ in the AD group ( $p=0.969)$ and from $15 \mathrm{~mL}(5-25)$ to $5 \mathrm{~mL}(5-20)$ $(\mathrm{p}=0.493)$ in the oPEP group. In the QoL-B domains, Vitality improved from a median (IQR) of 44 (27.566.8 ) to 67 (44-67) p=0.027, and Social Functioning from 50 (21-67) to 58 (37.5-76.5), p=0.042 in the

\section{TABLE 2 Effects of treatment on symptoms and lung function}

\begin{tabular}{|c|c|c|c|}
\hline & $A D$ & OPEP & p-value \\
\hline Subjects $n$ & 25 & 24 & \\
\hline Change in sputum purulence ${ }^{\#} \mathrm{n}(\%)$ & & & $>0.99$ \\
\hline Unchanged & $13(65)$ & $13(59)$ & \\
\hline Worsened & $3(15)$ & $4(18)$ & \\
\hline Change in sputum quantity $\mathrm{n}(\%)$ & & & 0.044 \\
\hline Change in sputum quantity $(\mathrm{mL})$; median (range) randomisation to end of study & $17.5(5-42.5)$ to $15(5-45)$ & $15(5-25)$ to $5(5-20)$ & 0.386 \\
\hline $\mathrm{LCl}$ change after 4 weeks ${ }^{4} \mathrm{n}(\%)$ & & & 0.847 \\
\hline Improved & $8(32)$ & $6(25)$ & \\
\hline Unchanged & $7(28)$ & $8(33)$ & \\
\hline Worsened & $10(40)$ & $10(42)$ & \\
\hline $\mathrm{FEV}_{1} \%$ change after 4 weeks, median (range) & $0.05(-23-7.6)$ & $0(-11.8-10.5)$ & 0.71 \\
\hline
\end{tabular}




\section{TABLE 3 Effects on quality of life}

\begin{tabular}{|c|c|c|c|c|c|c|}
\hline \multirow[t]{2}{*}{ QoL domain } & \multicolumn{3}{|c|}{$A D$} & \multicolumn{3}{|c|}{ OPEP } \\
\hline & Baseline & 4 weeks & $p$-value & Baseline & 4 weeks & $\mathrm{p}$-value \\
\hline Subjects $\mathrm{n}$ & 25 & & & 24 & & \\
\hline Respiratory symptoms & $67(44-79.5)$ & $70(50-79.5)$ & 0.732 & $65(46-77)$ & $72(48.8-80.3)$ & 0.139 \\
\hline Physical functioning & $60(20-83.5)$ & $67(37-86.5)$ & 0.393 & $53.5(21.8-93)$ & $60(36.5-87)$ & 0.412 \\
\hline Vitality & $44(27.5-66.8)$ & $67(44-67)$ & 0.027 & $44(1-100)$ & $50(33-67)$ & 0.231 \\
\hline Role functioning & $73(53-87)$ & $87(56.5-93)$ & 0.073 & $70(41.8-87)$ & $73(48.5-80)$ & 0.970 \\
\hline Emotional functioning & $83(54-100)$ & $83(62.5-100)$ & 0.611 & $79(60.3-92)$ & $79(67-98)$ & 0.690 \\
\hline Social functioning & $50(21-67)$ & $58(37.5-76.5)$ & 0.042 & $\mathrm{n}=2358(42-83)$ & $\mathrm{n}=2358(44-75)$ & 0.919 \\
\hline Treatment burden & $\mathrm{n}=2056(33-78)$ & $\mathrm{n}=2044(33-64.3)$ & 0.085 & $\mathrm{n}=2056(35.8-75.3)$ & $n=2056(33.3-67)$ & 0.458 \\
\hline Health perceptions & $42(33-67)$ & $58(42-75)$ & 0.177 & $33(25-58)$ & $42(33-64.8)$ & 0.048 \\
\hline
\end{tabular}

AD group. In the oPEP group, the score for Health Perceptions improved from 33 (25-58) to 42 (3364.5), $\mathrm{p}=0.048$ (table 3). Treatment burden score in the QoL-B did not change for the entire cohort but was worsened or unchanged in 17 of 20 participants (85\%) who reported at least $70 \%$ adherence in the $\mathrm{AD}$ group, and in only 11 of 20 (55\%) in the oPEP group ( $\mathrm{p}=0.038)$.

Spirometry did not change in either group (table 2). There was no association between 33\% or more sputum volume reduction and LCI change of 0.5 or more $(\mathrm{p}=0.532)$; sputum change and increase of 8 or more units in QoL-respiratory domain $(\mathrm{p}=0.770)$; and an increase of 8 or more units in QoL-respiratory domain and LCI change of 0.5 or more ( $\mathrm{p}=0.371$ ). 8 units is the established minimally clinically important difference (MCID) in the QoL-B [33]. To the best of our knowledge, the MCID for sputum volume was not established; 33\% was arbitrarily chosen to reflect a significant reduction in sputum volume.

\section{Discussion}

This study was designed to test the effects of two airway clearance methods in bronchiectasis on LCI and other outcome measures. We compared two established airway clearance methods: AD and oPEP, practised daily for 4 weeks. After 4 weeks, there was no difference in LCI between treatment groups, and LCI did not change significantly between visits in either group. The minimally important difference in LCI is not well established; we chose a low cut-off of 0.5 , which was the difference between groups reported in an intervention with inhaled hypertonic saline in children with CF [10]. Self-reported daily sputum quantity was reduced compared to baseline in significantly more patients randomised to oPEP than to AD (52\% versus $24 \%$, respectively; $\mathrm{p}=0.04$ ). Improvements in various domains of the QoL-B were seen in both groups. In contrast to studies investigating the benefits of a single airway clearance session, in which an increase in expectorated sputum was desired, we considered a reduction in 24-hour sputum quantity to reflect less inflammation and a beneficial outcome in bronchiectasis. A reduction in sputum volume was seen in several studies of long-term treatments in bronchiectasis [34-40], including mannitol, a mucoactive agent $[34,35]$. The recruited bronchiectasis patients were either newly diagnosed or referred. However, according to local practice in our centre, the majority of participants in both groups (73\% and $80 \%)$ started treatment with inhaled hypertonic saline prior to study inclusion. Hypertonic saline inhalation itself may decrease sputum purulence [41] and promote sputum clearance by coughing. Therefore, some effect of treatment may have occurred prior to randomisation to the study, and this may have lessened the treatment effect observed in the study.

The difference between these two airway clearance methodologies may reflect the efficacy in clearing sputum from the airways. However, training to perform AD may require more time than for oPEP. Since the study protocol limited training to a single session with the study therapist, the oPEP results may reflect the ease of training participants in self-managing their sputum production. Treatment burden was worsened or unchanged in $55 \%$ oPEP versus $85 \%$ of $\mathrm{AD}(\mathrm{p}=0.038)$ participants who reported adherence to daily airway clearance of $70 \%$ or above. The better results obtained by the oPEP group may reflect the ease of performing and persisting in using a self-managing device, in comparison to AD.

The LCI was not reduced by either method after 4 weeks of daily airway clearance. Similar to our findings, a previous study also did not find a good correlation between LCI and QoL in people with 
bronchiectasis [15]. In bronchiectasis, LCI is elevated compared to healthy controls [15]. This is believed to reflect lung inhomogeneity, with poorly ventilated areas contributing to delayed clearance of inert gas. Elevated LCI in bronchiectasis may be explained by mucus plugs obstructing and delaying gas mixture from portions of the bronchial tree, and clearance of mucus is hypothesised to improve LCI [31]. However, LCI was not found to significantly improve after a single airway clearance session [14, 23]. In CF, LCI was found to be responsive to long-term treatment with inhaled hypertonic saline, with a reduction of -0.63 units in preschool children [11], and is increasingly used as an end-point in clinical trials. To the best of our knowledge, LCI has never been reported to respond to a long-term intervention in adults with bronchiectasis, although evaluated as an exploratory end-point in a randomised trial of inhaled tobramycin [42, 43]. In contrast to studies of long-term interventions in CF, we did not find that LCI improves significantly after 4 weeks of airway clearance. This may reflect a difference between bronchiectasis patients, in whom changes to lung structure may be irreversible, from younger patients with CF, or may result from insufficient effect of physiotherapy. It will be interesting to see the effect on LCI by other bronchiectasis interventions.

Our study has some limitations. First, this study may have been under-powered to detect a change in LCI, as power analysis was based on cross-sectional studies of LCI in bronchiectasis, rather than response to treatment [13-15]. Second, we included patients with a relatively preserved lung function. This was intentional, as LCI performed better in people with preserved lung function, but may limit the extension of our results to more severely affected patients. The study design of 4 weeks, and once daily airway clearance, may not have been extensive enough to see changes in lung ventilation. While researchers were blinded to treatment allocations, participants were not, and this may have potentially affected results (mainly QoL-B but also sputum quantity). Finally, estimation of adherence and sputum volume were done by questioning rather than weighing, and thus may be subject to bias.

Despite the above limitations, to the best of our knowledge, this is the first study to compare the long-term effects of airway clearance on LCI. Future studies testing the responsiveness of LCI to therapeutic interventions in bronchiectasis are needed.

Acknowledgements: We thankfully acknowledge the assistance of Amir Shama and Amer Ubeid from the Pulmonology Institute, Carmel Medical Center, Haifa, Israel for performing spirometry, and of Sharon Avital and Esther Schneider from the Pulmonology Institute, Carmel Medical Center, Haifa, Israel for assistance conducting the clinical research. This work was previously presented at the 2020 ERS International Congress.

Provenance: Submitted article, peer reviewed.

Ethics approval: The study protocol was approved by Carmel Medical Center Helsinki committee (approval number CMC-87-16). All participants gave written informed consent before data collection began.

This study is registered at www.clinicaltrials.gov with identifier number NCT03013452. Data will be shared with researchers who provide a methodologically sound proposal.

Author contributions: M. Shteinberg had full access to all the data in the study, and takes responsibility for the integrity of the data and the accuracy of the data analysis, including and especially any adverse effects. G. Livnat participated equally in study design, interpretation of lung clearance indices, analysis of results and drafting the manuscript. N. Yaari, N. Stein, M. Hanna, M. Harel, L. Bentur, and Y. Adir contributed substantially to the study design, data analysis and interpretation, and the writing of the manuscript.

Conflict of interest: G. Livnat has nothing to disclose. N. Yaari has nothing to disclose. N. Stein has nothing to disclose. L. Bentur has nothing to disclose. M. Hanna has nothing to disclose. M. Harel has nothing to disclose. Y. Adir has nothing to disclose. M. Shteinberg reports grants from Trudell Pharma during the conduct of the study; and grants, personal fees and nonfinancial support from GSK, grants and personal fees from Novartis, personal fees from Boehringer Ingelheim, AstraZeneca, Kamada, Vertex Pharmaceuticals and Teva, and nonfinancial support from Actelion and Rafa, outside the submitted work; and is a member of the EMBARC group.

Support statement: Trudell Medical International (London, Canada) provided Aerobika devices and financial support, but did not influence the study design or interpretation of results. Funding information for this article has been deposited with the Crossref Funder Registry. 
References

1 Flume PA, Chalmers JD, Olivier KN. Advances in bronchiectasis: endotyping, genetics, microbiome, and disease heterogeneity. Lancet 2018; 392: 880-890.

2 Shteinberg M, Flume PA, Chalmers JD. Is bronchiectasis really a disease? Eur Respir Rev 2020; 29: 190051.

3 Polverino E, Goeminne PC, McDonnell MJ, et al. European Respiratory Society guidelines for the management of adult bronchiectasis. Eur Respir J 2017; 50: 1700629.

4 Spinou A, Chalmers JD. Respiratory physiotherapy in the bronchiectasis guidelines: is there a loud voice we are yet to hear? Eur Respir J 2019; 54: 1901610.

5 Murray MP, Pentland JL, Hill AT. A randomised crossover trial of chest physiotherapy in non-cystic fibrosis bronchiectasis. Eur Respir J 2009; 34: 1086-1092.

6 Figueiredo PHS, Zin WA, Guimarães FS. Flutter valve improves respiratory mechanics and sputum production in patients with bronchiectasis. Physiother Res Int 2012; 17: 12-20.

7 Smith MP, Hill AT. Evaluating success of therapy for bronchiectasis: what end points to use? Clin Chest Med 2012; 33: 329-349.

8 Robinson PD, Latzin P, Verbanck S, et al. Consensus statement for inert gas washout measurement using multiple- and single-breath tests. Eur Respir J 2013; 41: 507-522.

9 Rodriguez Hortal MC, Nygren-Bonnier M, Hjelte L. Non-invasive ventilation as airway clearance technique in cystic fibrosis. Physiother Res Int 2017; 22: e1667.

10 Stahl M, Wielpütz MO, Ricklefs I, et al. Preventive inhalation of hypertonic saline in infants with cystic fibrosis (PRESIS): a randomized, double-blind, controlled study. Am J Respir Crit Care Med 2019; 199: 1238-1248.

11 Ratjen F, Davis SD, Stanojevic S, et al. Inhaled hypertonic saline in preschool children with cystic fibrosis (SHIP): a multicentre, randomised, double-blind, placebo-controlled trial. Lancet Respir Med 2019; 7: 802-809.

12 Grosse-Onnebrink J, Mellies U, Olivier M, et al. Chest physiotherapy can affect the lung clearance index in cystic fibrosis patients. Pediatr Pulmonol 2017; 52: 625-631.

13 Gonem S, Scadding A, Soares M, et al. Lung clearance index in adults with non-cystic fibrosis bronchiectasis. Respir Res 2014; 15: 59.

14 Grillo L, Irving S, Hansell DM, et al. The reproducibility and responsiveness of the lung clearance index in bronchiectasis. Eur Respir J 2015; 46: 1645-1653.

15 Rowan SA, Bradley JM, Bradbury I, et al. Lung clearance index is a repeatable and sensitive indicator of radiological changes in bronchiectasis. Am J Respir Crit Care Med 2014; 189: 586-592.

16 Horsley A. Lung clearance index in the assessment of airways disease. Respir Med 2009; 103: 793-799.

17 Fuchs SI, Gappa M, Eder J, et al. Tracking Lung Clearance Index and chest CT in mild cystic fibrosis lung disease over a period of three years. Respir Med 2014; 108: 865-874.

18 Owens CM, Aurora P, Stanojevic S, et al. Lung Clearance Index and HRCT are complementary markers of lung abnormalities in young children with CF. Thorax 2011; 66: 481-488.

19 Trudell Medical International. AEROBIKA* OPEP Device. www.trudellmed.com/products/aerobika-opep-device Date last accessed: 24 April 2020.

20 Svenningsen S, Paulin GA, Sheikh K, et al. Oscillatory positive expiratory pressure in chronic obstructive pulmonary disease. COPD 2016; 13: 66-74.

21 Burudpakdee C, Near AM, Huang $\mathrm{H}$, et al. A real-world evidence study assessing the impact of adding the Aerobika oscillating positive expiratory pressure device to standard of care upon healthcare resource utilization and costs in post-operative patients. Pulm Ther 2018; 4: 87-101.

22 Pryor JA. Physiotherapy for airway clearance in adults. Eur Respir J 1999; 14: 1418-1424.

23 Poncin W, Reychler G, Leeuwerck N, et al. Short-term effect of autogenic drainage on ventilation inhomogeneity in adult subjects with stable non-cystic fibrosis bronchiectasis. Respir Care 2017; 62: 524-531.

24 O'Neill K, O'Donnell AE, Bradley JM. Airway clearance, mucoactive therapies and pulmonary rehabilitation in bronchiectasis. Respirology 2019; 24: 227-237.

25 Lee AL, Burge AT, Holland AE. Airway clearance techniques for bronchiectasis. Cochrane Database Syst Rev 2015; 11: CD008351.

26 Quittner AL, Marciel KK, Salathe MA, et al. A preliminary quality of life questionnaire-bronchiectasis: a patient-reported outcome measure for bronchiectasis. Chest 2014; 146: 437-448.

27 Hill AT, Haworth CS, Aliberti S, et al. Pulmonary exacerbation in adults with bronchiectasis: a consensus definition for clinical research. Eur Respir J 2017; 49: 1700051.

28 Murray MP, Pentland JL, Turnbull K, et al. Sputum colour: a useful clinical tool in non-cystic fibrosis bronchiectasis. Eur Respir J 2009; 34: 361-364.

29 McKane M, Crichton M, Dicker A, et al. Validation of a four point sputum colour chart in chronic obstructive pulmonary disease. Eur Respir J 2015; 46: PA3349.

30 Fuchs SI, Sturz J, Junge S, et al. A novel sidestream ultrasonic flow sensor for multiple breath washout in children. Pediatr Pulmonol 2008; 43: 731-738.

31 Kent L, Reix P, Innes JA, et al. Lung clearance index: evidence for use in clinical trials in cystic fibrosis. J Cyst Fibros 2014; 13: 123-138. 
Miller MR, Hankinson J, Brusasco V, et al. Standardisation of spirometry. Eur Respir J 2005; 26: 319-338.

Quittner AL, O'Donnell AE, Salathe MA, et al. Quality of Life Questionnaire-Bronchiectasis: final psychometric analyses and determination of minimal important difference scores. Thorax 2015; 70: 12-20.

Bilton D, Tino G, Barker AF, et al. Inhaled mannitol for non-cystic fibrosis bronchiectasis: a randomised, controlled trial. Thorax 2014; 69: 1073-1079.

Bilton D, Daviskas E, Anderson SD, et al. Phase 3 randomized study of the efficacy and safety of inhaled dry powder mannitol for the symptomatic treatment of non-cystic fibrosis bronchiectasis. Chest 2013; 144 215-225.

Wilson R, Welte T, Polverino E, et al. Ciprofloxacin dry powder for inhalation in non-cystic fibrosis bronchiectasis: a phase II randomised study. Eur Respir J 2013; 41: 1107-1115.

Stockley R, De Soyza A, Gunawardena K, et al. Phase II study of a neutrophil elastase inhibitor (AZD9668) in patients with bronchiectasis. Respir Med 2013; 107: 524-533.

Haworth CS, Foweraker JE, Wilkinson P, et al. Inhaled colistin in patients with bronchiectasis and chronic Pseudomonas aeruginosa infection. Am J Respir Crit Care Med 2014; 189: 975-982.

Lourdesamy Anthony Al, Muthukumaru U. Efficacy of azithromycin in the treatment of bronchiectasis. Respirology 2014; 19: 1178-1182.

40 Watz H, Nagelschmitz J, Kirsten A, et al. Safety and efficacy of the human neutrophil elastase inhibitor BAY 85-8501 for the treatment of non-cystic fibrosis bronchiectasis: a randomized controlled trial. Pulm Pharmacol Ther 2019; 56: 86-93.

1 Ramsey KA, Chen ACH, Radicioni G, et al. Airway mucus hyperconcentration in non-cystic fibrosis bronchiectasis. Am J Respir Crit Care Med 2020; 201: 661-670.

Loebinger MR, Polverino E, Blasi F, et al. Efficacy and safety of tobramycin inhalation powder in bronchiectasis patients with P. aeruginosa infection: design of a dose-finding study (iBEST-1). Pulm Pharmacol Ther 2019; 58: 101834.

O'Neill K, Ferguson K, Cosgrove D, et al. Multiple breath washout in bronchiectasis clinical trials: is it feasible? ERJ Open Res 2020; 6: 00363-2019. 